\title{
United States COVID-19 Vaccination Preferences (CVP): 2020 Hindsight
}

\author{
Benjamin Matthew Craig ${ }^{1}$
}

Accepted: 28 February 2021 / Published online: 30 March 2021

(c) The Author(s), under exclusive licence to Springer Nature Switzerland AG 2021

\begin{abstract}
Background Shortly after the 2020 US election, initial evidence on first-generation COVID-19 vaccines showed 70-95\% efficacy and minimal risks. Yet, many US adults expressed reluctance.

Aims The aim of this study was to compare persons willing and unwilling to be vaccinated against COVID-19 and to estimate the effects of vaccination attributes on uptake: proof of vaccination, vaccination setting, effectiveness, duration of immunity, and risk of severe side effects.

Method Between 9 and 11 November 2020, 1153 US adults completed a discrete choice experiment (DCE) on Phase 2 of the CDC Vaccination Program (August 2021). Each of its eight choice tasks had three vaccination alternatives and "no vaccination for 6 months." An opt-out inflated logit model was estimated to test for respondent differences and attribute effects. Results Respondent demographics were unrelated to one's willingness to be vaccinated ( $p$ value 0.533 ), but those with less education were more likely to be unwilling $(p<0.001)$. Among those willing, uptake ranged from 61.70 to $97.75 \%$, depending on the vaccination attributes. Effectiveness and safety had the largest effects. Offering proof of vaccination and a choice of setting increased uptake as much as increasing immunity from 3 to 6 months.

Conclusions To maximize uptake, the CDC Program should standardize proof of vaccination and offer a choice of setting, instead of a one-size-fits-all approach. If the first-generation vaccines are efficacious, widely available, and free, overall predicted uptake is $68.81 \%$ by the end of Phase 2 (August 2021), which is well below the $75-90 \%$ needed for herd immunity. Further health preference research is necessary to uncover and address unwillingness and reluctance to vaccinate against COVID-19.
\end{abstract}

\section{Key Points}

The CDC COVID-19 Vaccination Program should standardize proof of vaccination, namely the delivery of vaccination cards that can serve as verifiable receipts.

US adults also want a choice of setting, instead of a onesize-fits-all approach.

Even if the first-generation vaccines are efficacious, widely available, and free, predicted uptake is $68.81 \%$ by the end of Phase 2 (August 2021), which is well below $75-90 \%$ needed for herd immunity prior to the 20212022 influenza season.

Benjamin Matthew Craig

bcraig@usf.edu

1 Department of Economics, University of South Florida, CMC206A, 4202 E. Fowler Avenue, Tampa, FL 33620, USA

\section{Introduction}

The 2019 coronavirus (COVID-19) pandemic has affected the lives and livelihoods of nearly everyone around the world. In the United States, many currently have high hopes for the first-generation vaccines. However, "bringing a vaccine to market is only half the challenge; also critical is ensuring a high enough vaccination rate to achieve herd immunity" [1]. This health preference study seeks to understand the demand side of this directive, specifically, who is unwilling to be vaccinated against COVID-19 and the effects of a vaccination's attributes on its uptake among the willing.

During the initial wave of the pandemic, between 14 and 18 May, 2020, the Associated Press and the National Opinion Research Center (AP-NORC) at the University of Chicago asked 1056 US adults the following: "If a vaccine against the coronavirus becomes available, do you plan to get vaccinated, or not?" [2]. According to their results, roughly half of American adults (49\%) reported that they would get a COVID-19 vaccine if scientists working to produce one 
succeeded, $20 \%$ of those surveyed would refuse the vaccine, while $31 \%$ were not sure if they would get vaccinated. Other subsequent polls found similar results (Table 1), but polls do not fully capture vaccination reluctance or the causal relationship between vaccination attributes and uptake. Clearly, more evidence was needed to prepare the Centers for Disease Control and Prevention (CDC) COVID-19 Vaccination Program. This gap was originally identified during the June COVID-19 Health Preference Research (HPR) Roundtable, an open, monthly forum of scientists from around the world who are actively collaborating on COVID-19 HPR studies (see protocol), which also led to this themed issue [3].

This November 2020 study was specifically modelled after the CDC COVID-19 Vaccination Program Interim Playbook for Jurisdiction Operations version 2.0, which was published on October 29, 2020 [4]. A similar study was conducted by Kreps and colleagues in July 2020, well before the first-generation vaccines were known to be efficacious and such plans were disseminated [5]. Between May and November 2020, many noteworthy health preference studies examined various aspects of COVID-19 in the United States [6] and in other countries [7-13]. Hopefully, many more studies will follow, building an evidentiary basis to inform the development of public health programs [14].

With each COVID-19 HPR study, it is important to acknowledge its hypothetical scenario and historical context, including survey timing and sample characteristics. This study is based on a Phase 2 scenario and was conducted between November 9 and 11, 2020, a week after the US election. At that time, the US was experiencing over 1000 COVID-19 deaths per day [15]; the survey was administered after the announcement of initial evidence on firstgeneration COVID-19 vaccines showed 70-95\% efficacy, but before the availability of any vaccines (prior to Phase 1) [16]. To assess vaccination preferences expeditiously, US adults were recruited from a marketing panel (Dynata ${ }^{\circledR}$ ) using quotas, similar to prior US studies [17]. Although the target population may be considered geographically and demographically representative, its sample, like those of other online surveys, underrepresents persons in the lower quintile of educational attainment, such as persons who are illiterate or lack training with computers or the internet. Like with the polling evidence, the reader should account for the dynamic context and source when evaluating its findings.

Based on the May 2020 poll and more recent results (Table 1), support for COVD-19 vaccination is far from unanimous among the US general population, particularly among those at low risk. This November 2020 study contributes to our understanding by testing the association between respondent characteristics and the likelihood of being unwilling to be vaccinated against COVID-19, and by testing the causal relationships between the vaccination's attributes and uptake among those who are willing.

\section{Methods}

\subsection{What is Vaccination Preference Research?}

Vaccination preference research is the subfield of HPR dedicated to understanding the value of vaccinations, including vaccines and their services, outcomes, and supporting systems. In this paper, 'uptake' refers to a person's initial utilization of a vaccination service (either as a stated or revealed preference). In economics, uptake is a choice that defines the value of vaccinations from the perspective of its consumers.

Table 1 COVID-19 vaccination preference by critical populations ${ }^{\mathrm{a}}$

\begin{tabular}{|c|c|c|c|c|c|c|}
\hline & All & Essential workers ${ }^{b}$ & At increased risk & $\begin{array}{l}\text { Limited access to } \\
\text { vaccinations }\end{array}$ & Non-critical & $p$ value \\
\hline All & $100(1153)$ & $16.05(185)$ & $29.75(343)$ & $2.69(31)$ & $51.52(594)$ & \\
\hline \multicolumn{7}{|c|}{ If a vaccine against the coronavirus becomes available, do you plan to get vaccinated, or not? } \\
\hline No & $16.57(191)$ & $21.08(39)$ & $7.58(26)$ & $22.58(7)$ & $20.03(119)$ & \multirow[t]{3}{*}{$<0.001$} \\
\hline Don't know/not sure & $22.38(258)$ & $17.30(32)$ & $17.49(60)$ & $9.68(3)$ & $27.44(163)$ & \\
\hline Yes & $61.06(704)$ & $61.62(114)$ & $74.93(257)$ & $67.74(21)$ & $52.53(312)$ & \\
\hline \multicolumn{7}{|c|}{ In the case that you decide to be vaccinated, do you prefer to get a vaccination card, or not? } \\
\hline No vaccination card & $8.95(63)$ & $12.28(14)$ & $6.23(16)$ & $0.00(0)$ & $10.58(33)$ & \multirow[t]{2}{*}{0.073} \\
\hline Vaccination card & $91.05(641)$ & $87.72(100)$ & $93.77(241)$ & $100.00(21)$ & $89.42(279)$ & \\
\hline \multicolumn{7}{|c|}{ In the case that you decide to be vaccinated, do you prefer to be vaccinated in a medical or community setting? } \\
\hline Community setting & $25.28(178)$ & $27.19(31)$ & $24.51(63)$ & $61.90(13)$ & $22.76(71)$ & \multirow[t]{2}{*}{0.001} \\
\hline Medical setting & $74.72(526)$ & $72.81(83)$ & $75.49(194)$ & $38.10(8)$ & $77.24(241)$ & \\
\hline
\end{tabular}

Data is presented as $\%(n)$

${ }^{a}$ The critical population definitions were taken from the CDC and based on self-report

${ }^{\mathrm{b}}$ This includes healthcare personnel and other essential workers in paid or unpaid positions 
In the theoretical framework of this paper, all individuals are described by their likelihood of belonging to one of two groups (i.e., grade of membership): individuals who are unwilling to be vaccinated against COVID-19 (non-traders) and individuals who are willing to be vaccinated (traders). By definition, individuals unwilling to be vaccinated will not utilize Phase 2 vaccination services. Such non-traders may be unwilling for a variety of reasons unrelated to COVID-19 or its vaccines, such as a lack of health literacy or education, or a general distrust of science and government (see Protocol in the electronic supplementary material [ESM] for discussion on anti-vaxxers).

Among those willing to be vaccinated against COVID19 in Phase 2 (i.e., traders), a vaccine is a good that would be accepted gladly under certain conditions (i.e., market demand). However, shortfalls in vaccination attributes may induce reluctance. Mounting reluctance may cause hesitance, a behavioral precursor impeding the uptake of a recommended vaccine. Uptake among non-traders is deterministic ('hard no') and uptake among traders is probabilistic (McFadden's positivity axiom) [18]. In this study, US adults chose between alternative vaccinations under a common Phase 2 scenario, revealing their grade of membership and the effects of vaccination attributes on uptake simultaneously.

\subsection{Descriptive Framework}

The descriptive framework delineates the hypothetical scenario of the decision context and the vaccination attributes (Protocol, see ESM). It was largely taken from the published literature on other vaccinations $[19,20]$, expert consultations [3], and the CDC interim playbook version 2.0 [4].

In this hypothetical scenario, each respondent witnessed the persistence of the COVID-19 pandemic at the start of 2021 with new cases and preventable deaths being reported each day. Although multiple vaccines have been approved by the US Food and Drug Administration (FDA) and the $\mathrm{CDC}$, these first-generation vaccines were in limited supply initially (Phase 1), and their doses were distributed in a limited manner, focusing on critical populations as defined by the CDC.

After Phase 1, the COVID-19 vaccines became widely available and free for the general population. The effectiveness and safety of the first-generation vaccines were well established based on Phase 1 evidence. At the start of Phase 2, the CDC recommended that all US adults be vaccinated (two injections, 1 month apart). In this decision context, the respondent has not been vaccinated yet and must choose between alternative vaccinations against COVID-19 or "no vaccination for six months," skipping the Phase 2 availability of the first generation of vaccines. While other vaccines are under development, no other vaccines are expected to be approved and available within 6 months.

Each vaccination is described using five attributes (Table 3; Protocol, see ESM). The proof of vaccination, vaccination setting, and duration of immunity are described using adjectival statements. The two risk attributes, effectiveness and risk of severe side effects, are expressed numerically and using graphics, namely skyline images [21] and icon arrays [22]. All 64 alternative vaccinations in the descriptive framework are free and incur a risk of mild side effects similar to an influenza vaccination.

\subsection{Experimental Design}

The design of the discrete choice experiment (DCE) assigns a series of eight choice sets to each respondent (Protocol, Screenshots, see ESM). Overall, the design is composed of three design matrices, each with 56 unique sets (Experimental design spreadsheet; seven blocks of eight sets, see ESM). For each matrix, a different method was used for set selection (random, generator-developed, and efficient) to allow design comparison in future research. Unlike the random and generator-developed designs, the efficient design was optimized based on a conditional logit model, $D$-error, and fixed priors for the coefficients $\beta=(0,0,0.2,0.2,0.1,0.2$, $0.3)$ under the assumption of preference homogeneity and excluding the opt-out [23]. Coverage of each design matrix was evaluated based on uniqueness (sets, objects, pairs, responses), frequency of trade-offs, and multicollinearity under multiple potential scenarios including utility balance (unweighted), priors, and five times the priors (Appendix 3, see ESM). At least fifty unique respondents completed each of the 168 sets in concordance with the De Moivre's NP5 rule [24].

\subsection{Survey Instrument}

The survey instrument (see screenshots in the ESM) has five active components (consent, screener, background, choice tasks, and follow-up) and two passive components (panel recruitment and paradata) [25]. It was constructed to collect one to three responses per page and to show a progression bar with the proportion of pages remaining. The questions were also numbered (1 consent, 5 screener, 10 background, 12 choice tasks, and 30 follow-up questions). Respondents could return to previous pages, except between sections and tasks due to randomization and adaptive features. Respondents could not return to the survey once the browser had been closed.

The screener informed the quota recruitment, and the background section was designed to introduce the respondents to the five attributes, to elicit their preferences on nominal attribute levels (proof of vaccination and vaccination 
setting; Table 1), and to capture their predictions about firstgeneration vaccines (Protocol and Screenshots, see ESM). Some aspects of the instrument are novel, and others built from past studies. For example, the effectiveness vignette was once used in an influenza vaccination study [19] and the serious game was once used to extend risk descriptions [21] in a meningitis vaccination study [20].

Prior to the initial eight choice tasks (i.e., triplets with opt-out), the warm-up task (Fig. 1) included two dominated alternatives [26] and was followed by a confirmatory task with an honesty oath [27]. Like the consent form, each respondent was required to demonstrate task performance and confirm four statements, indicating a minimum understanding of the study purpose, the hypothetical scenario, and the implications of opt-out (see Screenshots in the ESM). The subsequent eight tasks and the attribute order were randomized at the subject level (i.e., different between subjects, same across tasks). Positions of the three vaccination alternatives (i.e., left-right) were randomized at the task level keeping the opt-out on the far right.

The choice task and descriptive framework were alphatested with HPR experts from the COVID-19 HPR Roundtable [3]. The survey instrument was beta-tested by ten HPR experts who provided written feedback and through structured interviews with nine anonymous US adults recruited through a popular crowdsourcing platform (Beta-testing spreadsheet) [28].

Prior to fielding, an independent review board (IRB; Advarra ${ }^{\circledR}$ ) reviewed the protocol and survey instrument. On 5 November 2020, the IRB determined this research project (Pro00047418) was exempt from IRB oversight based on the US Department of Health and Human Services regulations found at 45 CFR 46.104(d)(2). The study did not pose any physical risks, but there may be a risk of psychological distress resulting from questions that ask respondents to evaluate alternative scenarios relating to COVID-19.

\subsection{Fielding}

After the IRB exemption determination, US adults were recruited from a marketing panel (Dynata ${ }^{\circledR}$ ) targeting 18 demographic quotas (Protocol, see ESM) [29-31]. Under a double opt-in process, respondents consented when they first joined the panel and on the first page of the survey instrument. Two exclusion criteria were applied: (i) residing outside the 50 US states or the District of Columbia; and (ii) being age 17 years or less (18 years in Nebraska or Alabama or 20 in Mississippi). Apart from the consent and two exclusion criteria, no respondents were excluded from participation; however, participants could leave the survey

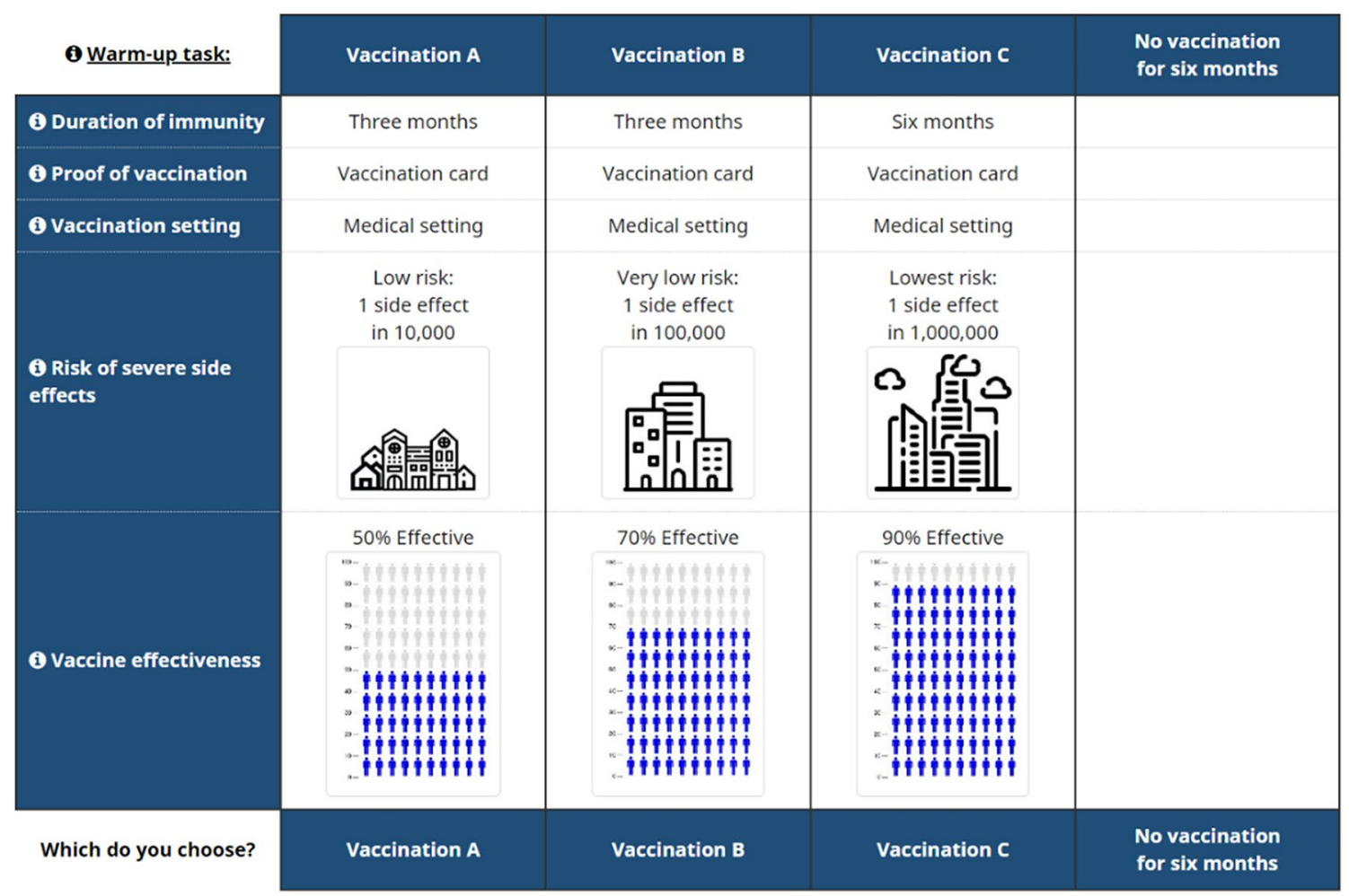

Fig. 1 Warm-up for the choice tasks with two dominated alternatives 
at any time by closing their internet browser or by leaving a comment (Appendix 1, see ESM).

After being screened, respondents were randomly assigned to one of the three designs and one of its seven blocks. Upon completion of their survey instrument, respondents were compensated using a point-based currency by the panel vendor. Data monitoring occurred daily and involved downloading the de-identified data to assess participation and revise quota recruitment.

\subsection{Econometric Analysis}

Once the fielding was completed, the analysis plan began with three steps (see Protocol, ESM): (i) descriptive analysis of respondent characteristics and participatory behaviors, characterizing external validity (Appendix 1, see ESM); (ii) descriptive analysis of response quality, characterizing internal validity of the DCE (Appendix 2, see ESM); and (iii) the primary analysis of the respondent differences and main effects using a modified version of a latent class logit (Tables 2 and 3).
Table 2 Respondent characteristics and their odds of being unwilling to be vaccinated against COVID-19

\begin{tabular}{|c|c|c|c|c|}
\hline \multirow[t]{2}{*}{ Respondent characteristics } & \multirow{2}{*}{$\begin{array}{l}\text { Sample } \\
100.00(1153) \\
\%(n)\end{array}$} & \multicolumn{3}{|c|}{$\begin{array}{l}\text { Unwilling to be vaccinated against } \\
\text { COVID-19 }\end{array}$} \\
\hline & & Odds ratio $^{a}$ & $\begin{array}{l}t \text { test } \\
p \text { value }\end{array}$ & $\begin{array}{l}\text { Wald } \\
p \text { value }\end{array}$ \\
\hline Age in years & & & & 0.268 \\
\hline $18-24$ & $6.16(71)$ & 0.529 & 0.359 & \\
\hline $25-34$ & $22.98(265)$ & 0.552 & 0.086 & \\
\hline $35-54$ & $38.68(446)$ & 1.000 & & \\
\hline $55-64$ & $8.07(93)$ & 1.016 & 0.968 & \\
\hline 65 and older & $24.11(278)$ & 0.54 & 0.099 & \\
\hline Gender & & & & 0.292 \\
\hline Male & $48.74(562)$ & 1.000 & & \\
\hline Female/other & $52.26(564)$ & 1.306 & 0.292 & \\
\hline Race & & & & 0.652 \\
\hline White alone & $76.67(884)$ & 1.000 & & \\
\hline Black or African American alone & $12.06(139)$ & 0.836 & 0.622 & \\
\hline Asian alone & $6.24(72)$ & 0.694 & 0.512 & \\
\hline Other & $5.03(58)$ & 1.523 & 0.408 & \\
\hline Ethnicity & & & & 0.488 \\
\hline Other & $87.25(1006)$ & 1.000 & & \\
\hline Hispanic or Latino & $12.75(147)$ & 1.265 & 0.488 & \\
\hline Educational attainment & & & & $<0.001$ \\
\hline High school or less & $14.57(168)$ & 1.000 & & \\
\hline Associates/some college & $24.63(284)$ & 0.626 & 0.122 & \\
\hline Bachelors or more & $60.80(701)$ & 0.306 & $<0.001$ & \\
\hline Critical populations & & & & 0.007 \\
\hline Non-critical & $54.20(625)$ & 1.000 & & \\
\hline Essential workers & $16.05(185)$ & 0.182 & 0.011 & \\
\hline At increased risk & $29.75(343)$ & 0.377 & 0.025 & \\
\hline COVID-19 diagnosis & & & & 0.201 \\
\hline Non-clinical & $85.08(981)$ & 1.000 & & \\
\hline Quarantined & $11.62(134)$ & 0.108 & 0.150 & \\
\hline Tested positive & $3.30(38)$ & 0.119 & 0.203 & \\
\hline
\end{tabular}

${ }^{\mathrm{a}}$ For the base case (a white, non-Hispanic, middle-aged, male adult who belongs to the non-clinical noncritical population and has a high school diploma or less), the odds of being unwilling is 0.353 (95\% CI $0.199-0.625$ ), which implies that at least $26.09 \%$ are unwilling to be vaccinated against COVID-19 regardless of the vaccination's attributes $(0.353 /(1+0.353))$. The probability of being unwilling drops significantly to $9.75 \%$ when the person receives a bachelor's degree or higher $(0.108 /(1+0.108))$ 
Table 3 The effect of vaccination attributes on uptake among those willing to be vaccinated against COVID-19

\begin{tabular}{|c|c|c|c|}
\hline 8 tasks for 1153 respondents & $\ln \left(\right.$ odds ratio) ${ }^{\mathrm{a}}$ & $95 \% \mathrm{CI}$ & $p$ value \\
\hline Vaccination (best-case scenario), $\beta_{0}$ & 3.773 & (3.079 to 4.467$)$ & $<0.001$ \\
\hline \multicolumn{4}{|l|}{ Proof of vaccination } \\
\hline $\begin{array}{l}\text { No vaccination card vs vaccination card, } \beta_{1} \\
\text { Vaccination setting }\end{array}$ & -0.318 & $(-0.254$ to -0.381$)$ & $<0.001$ \\
\hline Community vs medical setting, $\beta_{2}$ & -0.327 & $(-0.264$ to -0.390$)$ & $<0.001$ \\
\hline \multicolumn{4}{|l|}{ Vaccine effectiveness } \\
\hline $50 \%$ vs $70 \%$ effective, $\beta_{3}$ & -1.220 & $(-1.117$ to -1.324$)$ & $<0.001$ \\
\hline \multicolumn{4}{|l|}{ Duration of immunity } \\
\hline \multicolumn{4}{|l|}{ Risk of severe side effects ${ }^{b}$} \\
\hline \multicolumn{4}{|l|}{ Lowest risk: 1 side effect in $1,000,000$} \\
\hline vs Very low risk: 1 side effect in $100,000, \beta_{5}$ & -0.181 & $(-0.099$ to -0.264$)$ & $<0.001$ \\
\hline vs Low risk: 1 side effect in $10,000, \beta_{6}$ & -0.573 & $(-0.466$ to -0.680$)$ & $<0.001$ \\
\hline vs Moderate risk: 1 side effect in $1000, \beta_{7}$ & -0.982 & $(-0.850$ to -1.114$)$ & $<0.001$ \\
\hline
\end{tabular}

${ }^{a}$ In the best-case scenario (card, medical, 70\%, 6 months, lowest risk), predicted uptake is $97.75 \%$ (i.e., $\exp (3.773) /(1+\exp (3.773))$, where the constant $\beta_{0}$ equals 3.773). In the worst-case scenario, uptake drops to $61.70 \%\left(\exp (0.477) /(1+\exp (0.477))\right.$, where $\beta_{0}-\left(\beta_{1}+\beta_{2}+\beta_{3}+\beta_{4}+\beta_{7}\right)$ equals 0.477$)$

${ }^{\mathrm{b}}$ The coefficient estimates for the dummy variables show the effect of an increase in risk (i.e., lowest vs very low, lowest vs low, lowest vs moderate). The proportional risk assumption under expected utility theory (i.e., $\left.\beta_{5} / 9=\beta_{6} / 99=\beta_{7} / 999\right)$ was rejected $(p<0.001)$

\subsubsection{Latent Class Logit}

A latent class logit assumes that the population can be decomposed into $C$ distinct latent classes such that subjects of each class $(c=1, \ldots, C)$ are homogeneous with respect to their class-specific parameters $\beta_{c}$, which differ between the classes. To indicate that the choice probabilities depend on class membership $c$, the conditional logit model may be expressed as

$\operatorname{Pr}\left(y_{i j}=1 \mid c\right)=\frac{\exp \left(X_{i j} \beta_{c}\right)}{\sum_{k=1}^{J} \exp \left(X_{i k} \beta_{c}\right)}$,

and the probability of belonging to class $c$ conditionally on additional covariates $Z_{i}$ (i.e., grade-of-membership) can be expressed using a separate multinomial logit:

$\operatorname{Pr}(c)=\frac{\exp \left(Z_{i} \theta_{c}\right)}{\sum_{s=1}^{C} \exp \left(Z_{i} \theta_{s}\right)}$

This latent class model puts individuals into probabilistic groups based on their likelihood of belonging in that group; therefore, the probability density associated with individual $i$ has the following form:

Latent class logit : $\operatorname{Pr}\left(y_{i j}=1\right)$

$$
\begin{aligned}
& =\sum_{c=1}^{C} \operatorname{Pr}\left(y_{i j}=1 \mid c\right) \operatorname{Pr}(c) \\
& =\sum_{c=1}^{C} \frac{\exp \left(X_{i j} \beta_{c}\right)}{\sum_{k=1}^{J} \exp \left(X_{i k} \beta_{c}\right)} \frac{\exp \left(Z_{i} \theta_{c}\right)}{\sum_{s=1}^{C} \exp \left(Z_{i} \theta_{s}\right)} .
\end{aligned}
$$

\subsubsection{Opt-Out Inflated Logit}

Based on the theoretical framework, the opt-out inflated logit is a modified version of the latent class logit with only two classes (traders and non-traders). In this model, those unwilling to be vaccinated against COVID-19 (i.e., non-traders) do not choose vaccination, regardless of its attributes:

$\operatorname{Pr}\left(y_{i \text { optout }}=1 \mid\right.$ nontrader $)=1$ and

for $j \in\{1,2,3\}, \operatorname{Pr}\left(y_{i j}=1\right.$ |nontrader $)=0$.

Among the traders, the conditional $\log i t X_{i j} \beta$ describes the causal relationship between vaccination attributes and the likelihood of a choice. Apart from its constant, the model includes seven incremental dummy-coded variables, one for each of the first four two-level attributes and three for the last four-level attribute (risk of severe side effects).

The multinomial logit $Z_{i} \theta$ describes the grade of membership, namely its association with the respondent characteristics $Z_{i}, 15$ dummy-coded variables representing the respondent's age, gender, race, ethnicity, educational attainment, critical population, and COVID-19 status. These characteristics were selected based on the recruitment quotas and the CDC Vaccination Program definitions as self-reported by the respondents.

Overall, the opt-out inflated logit allows for heterogeneity in grade of membership (between-class) and homogeneity in vaccination preferences among traders (within-class), 


$$
\begin{aligned}
\operatorname{Pr}\left(y_{i j}=1\right)= & \operatorname{Pr}(\text { nontrader }) \operatorname{Pr}\left(y_{i j}=1 \mid \text { nontrader }\right) \\
& +\operatorname{Pr}(\text { trader }) \operatorname{Pr}\left(y_{i j}=1 \mid \text { trader }\right) .
\end{aligned}
$$

It inherently inflates the likelihood of choosing "no vaccination for 6 months" based on the respondent characteristics $Z_{i}$. Its 24 parameters and their $95 \%$ confidence intervals were estimated by maximum likelihood with respondent clusters, which accommodates the eight responses per respondent [32]. The significance of each coefficient was assessed using a t-test and their joint significance was assessed using a Wald test. For example, the Wald test was conducted to assess the proportional equivalence in three coefficients for the risk of severe side effects $\left(\beta_{5} / 9=\beta_{6} / 99=\beta_{7} / 999\right)$ as implied by expected utility theory.

After estimating the primary model, an exploratory analysis tested two interactions and two modifiers in the conditional logit. Effectiveness and duration of immunity were interacted, based on the expected utility theory (i.e., duration only matters if effective). Likewise, proof of vaccination and duration of immunity attribute were interacted because the duration of immunity may affect the card's expiry. Card preferences (Table 1) may modify the effects of vaccination cards (e.g., the effect of a card among those who want one versus those who do not) and setting preferences may modify the effect of vaccination setting (the effect of medical setting among those who prefer a medical setting versus those who prefer a community setting).

\section{Results}

\subsection{Respondent Characteristics}

Between 9 and 12 November 2020, 1529 US adults were recruited from a marketing panel and qualified as respondents (Appendix 1, see ESM). Some respondents (376; 25\%) dropped out during the survey; more older adults dropped out ( $p$ value $<0.001$ ). Drop-out was not associated with gender, race, or Hispanic ethnicity ( $p$ values of $0.158,0.502$, and 0.594 , respectively). The analytical sample (Table 2) differs from the US general population in terms of demographics and state of residence, but most differences were small $(<1 \%)$. Compared with the US 2019 American Community Survey, three noteworthy differences were the underrepresentation of Hispanic ethnicity $(-3.65 \%)$ and persons aged 55 years or older $(-5.64 \%)$, as well as a complete lack of respondents from South Dakota.

Apart from being demographically and geographically representative, the respondents in the analytical sample are more educated and have less household income compared with national estimates. In the sample, $60.80 \%$ (701) have a bachelor's degree or higher, approximately double the
$29.18 \%$ found nationally. Likewise, $14.57 \%$ (168) have a high school diploma or less, about half the $33.73 \%$ found nationally. Overall, this sample is not representative of persons in the lowest educational quintile due to its use of a marketing panel and online survey instrument.

\subsection{Experimental Design and Response Behaviors}

In terms of internal validity, the survey instrument successfully randomized the set assignment, attribute order, task sequence, and object positions, and these random components were not associated with the 18 demographic quotas (Appendix 2, see ESM). Median length of survey was 18 min and $40 \mathrm{~s} \mathrm{(IQR} \mathrm{790-1584} \mathrm{s).} \mathrm{Few} \mathrm{respondents} \mathrm{(2.78 \% ;}$ 32) selected the same vaccination alternative in all eight tasks, but $7.55 \%$ (87) selected opt-out consistently (potentially non-traders) and $76.76 \%$ (885) never selected opt-out. The likelihood of opt-out remained stable over the task sequence; however, the likelihood of choosing 'Vaccination A' increased slightly from 26.02 to $30.96 \%$ from the first to eighth task. Median response time for the eight choice tasks was $9.74 \mathrm{~s}$ (IQR 5.01-17.95 s) and decreased from $12.79 \mathrm{~s}$ in the first task to $8.17 \mathrm{~s}$ in the eighth task.

\subsection{COVID-19 Vaccination Preferences}

Table 1 shows the responses to three preference questions by self-reported population. Most respondents $(61.06 \%, 704)$ planned to be vaccinated; however, this was less prevalent in the non-critical population $(p<0.001)$. Among those who plan to be vaccinated, $91.05 \%$ (641) prefer to receive a vaccination card as proof of vaccination and $74.72 \%$ (526) prefer to be vaccinated in a medical setting. Unlike other critical populations, those who report limited access to vaccinations prefer to be vaccinated in a community setting. Such poll responses provide a quick snapshot of vaccination preferences generally, but poorly predict uptake. For example, $46.07 \%$ of those who do not plan to be vaccinated (88 out of 191) always chose vaccination in the eight tasks (Appendix 2, see ESM).

\subsection{Who is Unwilling To Be Vaccinated Against COVID-19?}

The likelihood of being unwilling to be vaccinated against COVD-19 (i.e., non-trader) has a median of $13.51 \%$ (IQR $6.61-21.28$ ), ranging from $0.95 \%$ to $35.80 \%$. Specifically, the likelihood for a non-Hispanic, White, male respondent aged 35-54 years who has a high school diploma or less, is not in a critical population, and has not been quarantined is $26.09 \%$ (95\% CI 16.62-38.46). The odds ratios for the demographic variables (Table 2, column 2) were insignificant $(p=0.533)$, but persons with more education, those 
at increased risk, and essential workers are less likely to be non-traders $(p<0.001)$.

\subsection{What Determines Uptake Among Those Willing to be Vaccinated?}

Across the 64 potential vaccinations described in this study, the likelihood of uptake has a median of $89.34 \%$ (IQR 83.16-94.38), ranging from $61.70 \%$ to $97.75 \%$. As hypothesized, each decrease in effectiveness, duration, or safety reduces the likelihood of uptake (Table 3; $p<0.001$ ). Reducing effectiveness from $70 \%$ to $50 \%$ had the largest effect, reducing the ln odds of uptake by 1.220 (95\% CI 1.117-1.324). Increasing the risk of severe side effects from 1 in 1,000,000 to 1 in 1000 reduced ln odds of uptake by 0.982 (95\% CI 0.850-1.114). Reducing duration of immunity caused a smaller reduction $(0.450 ; 95 \%$ CI 0.368-0.532). Contrary to expected utility theory, there was no significant interaction between effectiveness and duration $(p=0.890)$, and its proportional risk assumption (i.e., $\left.\beta_{5} / 9=\beta_{6} / 99=\beta_{7} / 999\right)$ was rejected $(p<0.001)$. However, the loss in value due to no vaccination card is 0.376 when duration is 6 months and 0.257 when it is 3 months $(p=0.026)$, suggesting that proof is more valuable when it lasts longer.

\subsection{Why Offer a Vaccination Card? Choice of Setting?}

'No vaccination card' reduces the ln odds of uptake by 0.383 (95\% CI 0.304-0.463) among those who want one, but does not affect uptake among those who do not $(0.049 ; 95 \%$ CI -0.160 to 0.258 ). Therefore, offering proof of vaccination, namely cards verifying vaccination status, produces an increase in uptake similar to extending duration of immunity from 3 to 6 months $(p=0.175)$.

Among those who prefer a medical setting, requiring a community setting reduces the ln odds of uptake by 0.481 (95\% CI 0.408-0.553). Conversely, requiring a medical setting reduces $\ln$ odds of uptake by 0.168 (95\% CI 0.043 , 0.286 ) among those who prefer a community setting. Offering a medical setting produces an increase in uptake similar to extending duration of immunity from 3 to 6 months among those who prefer the medical setting $(p=0.693)$.

\section{Discussion}

In summary, individuals who are unwilling to be vaccinated against COVID-19 tend to be less educated members of non-critical populations. Taking this diversity into account, the likelihood of uptake ranges from $36.60 \%$ to $61.11 \%$ under the worst-vaccination scenario $(61.70 \%)$ and from 62.75 to $96.82 \%$ under the best-vaccination scenario (97.75\%) among similar populations. A likely Phase 2 strategy will be to offer COVID-19 vaccination generally to achieve herd immunity potentially and expand educational opportunities and communication in regions that exhibit poor uptake. How to build sufficient trust in science and government programs in hard-to-reach populations is beyond the scope of this paper.

Among those willing to be vaccinated against COVID19, a vaccine's safety and effectiveness had the largest effects on uptake, which was not surprising. However, these attributes are typically beyond the control of a vaccination program. The findings also imply that the Phase 2 programs should offer a choice of setting and focus on communicating the availability and merit of having proof of vaccination. Vaccination cards may serve as standardized receipts and produce a meaningful increase in uptake. For example, some respondents during beta testing expressed that they do not care about the vaccine per se, but are willing to be vaccinated to get the card, given its potential privileges, its use as a status symbol, or as a ticket to normalcy.

This evidence is not easily compared with findings from the July 2020 study by Kreps and colleagues [5]. The July survey included five choice tasks (two alternatives with opt-out) with ancillary 7-point scales. Its descriptive framework is distinct in its historical context and includes vaccination and political attributes (e.g., endorsed by President Donald Trump). Methodological differences include a linear probability model, lack of graphics, and no description of opt-out timing (e.g., 'not to be vaccinated' versus 'no vaccination for 6 months'). Overall, the predicted uptake ranged between $40 \%$ and $71 \%$ in the July study (vaccination attributes only) and between $36.6 \%$ and $96.82 \%$ in the November study depending on the respondent characteristics and vaccination attributes. Both studies rely on marketing panels, which may explain their high predictions. Furthermore, any predictions on vaccination uptake may fail to account for future events, such as virus variants and peer effects, and be imprecise due to omitted attributes, variations in the model specification, and sampling limitations.

As demonstrated by polls during the 2020 US national election, online surveys have multiple biases in their predictions of individual behaviors. The focus of this study is testing associations and causal relationships, which may be less susceptible to limitations that affect prediction. Its results should be interpreted within their historical context and sampling. The development of the descriptive framework and survey instrument was based on expert consultations and the published literature, not formative 
qualitative research [33]. Graphics were included for the two probabilistic attributes, not the three deterministic attributes. A future analysis may examine heterogeneity in taste and scale among those willing to be vaccinated using more complex models that account for respondent characteristics, such as political affiliations and influenza vaccination behaviors.

\section{Conclusions}

From the perspective of the CDC COVID-19 Vaccination Program during Phase 2, this paper has four key lessons. First, persons in noncritical populations with a high school degree or less are more likely to be unwilling to be vaccinated, regardless of vaccination attributes. Second, proof of vaccination may be of great value to those willing to be vaccinated. Therefore, CDC should invest in and communicate a national standard for such cards for use by the general population. To some, a verifiable receipt in hand is a ticket to normalcy and demonstrates fulfillment of their civic duty (e.g., "I voted" sticker). Third, US adults want a choice of setting. Most prefer a medical setting and others prefer a community setting. Lastly, if the CDC program offers a vaccination card, a choice of setting, and the best vaccination hypothesized, this trifecta may render $86.02 \%$ uptake among similar populations by August 2021. However, this study cannot predict uptake among the lower quintile of educational attainment; therefore, a more realistic prediction is $68.81 \%$ in Phase $2(80 \% \times 86.02 \%)$, which is well below the high vaccine coverage (75-90\%) needed for herd immunity in the United States prior to the 2021-2022 influenza season [34].

Supplementary Information The online version contains supplementary material available at https://doi.org/10.1007/s40271-021-00508-0.

\section{Declarations}

Funding Benjamin M. Craig provided all financial support for the project.

Conflicts of interest/competing interest Benjamin M. Craig has no conflicts to disclose.

Availability of data and material The datasets generated during and analyzed during the current study are available from the corresponding author on reasonable request.

Acknowledgement BMC would like to thank the members of the COVID-19 HPR Roundtable and those who participated in the alphaand beta-testing of the preference-elicitation tasks andsurvey instrument, particularly his current and former graduate students: John D. Hartman, Stephen Poteet, Suzana Karim, and Maksat Jumamyradov.
Code availability The code generated used in the analysis the study datasets are available from the corresponding author on reasonable request.

Author contributions BMC developed the research question, methodology and design of the study as well as conducted the data analysis, interpreted the results, and wrote the final manuscript. BMC would like to thank the members of the COVID-19 HPR Roundtable and those who participated in the alpha- and beta-testing of the preferenceelicitation tasks and survey instrument, particularly his current and former graduate students: John D. Hartman, Stephen Poteet, Suzana Karim, and Maksat Jumamyradov

\section{References}

1. Mello MM, Silverman RD, Omer SB. Ensuring uptake of vaccines against SARS-CoV-2. N Engl J Med. 2020;383(14):1296-9. https://doi.org/10.1056/NEJMp2020926.

2. AP-NORC. The May 2020 AP-NORC Center Poll. Chicago: The Associated Press-NORC Center for Public Affairs Research, University of Chicago2020.

3. Craig BM, Sepúlveda JMG, Johnson FR, Kessels R, Reed SD, Sandorf E, et al. COVID-19 health preference research: four lessons learned. ISPOR Value Outcomes Spotlight. 2020;6(5):1-2.

4. US Centers for Disease Control and Prevention (CDC). COVID19 Vaccination Program Interim Playbook for Jurisdiction Operations, version 2.0: U.S. Department of Health and Human Services. 2020 October 29, 2020.

5. Kreps S, Prasad S, Brownstein JS, Hswen Y, Garibaldi BT, Zhang B, et al. Factors associated with US adults' likelihood of accepting COVID-19 vaccination. JAMA Netw Open. 2020;3(10):e2025594. https://doi.org/10.1001/jamanetworkopen.2020.25594.

6. Reed S, Gonzalez JM, Johnson FR. Willingness to accept tradeoffs among COVID-19 cases, social-distancing restrictions, and economic impact: a Nationwide US Study. Value Health. 2020;23(11):1438-43. https://doi.org/10.1016/j.jval.2020.07.003.

7. García LY, Cerda AA. Acceptance of a COVID-19 vaccine: a multifactorial consideration. Vaccine. 2020;38(48):7587. https:// doi.org/10.1016/j.vaccine.2020.10.026.

8. Chorus C, Sandorf ED, Mouter N. Diabolical dilemmas of COVID-19: an empirical study into Dutch society's trade-offs between health impacts and other effects of the lockdown. PLoS One. 2020;15(9):e0238683. https://doi.org/10.1371/journal.pone. 0238683.

9. Jonker M, de Bekker-Grob E, Veldwijk J, Goossens L, Bour S, Rutten-Van Mölken M. COVID-19 contact tracing apps: predicted uptake in the Netherlands based on a discrete choice experiment. JMIR Mhealth Uhealth. 2020;8(10):e20741. https://doi.org/10. 2196/20741.

10. Dong D, Xu RH, Wong EL-y, Hung C-T, Feng D, Feng Z et al. Public preference for COVID-19 vaccines in China: a discrete choice experiment. Health Expect. https://doi.org/10.1111/hex. 13140.

11. Degeling C, Chen G, Gilbert GL, Brookes V, Thai T, Wilson A, et al. Changes in public preferences for technologically enhanced surveillance following the COVID-19 pandemic: a discrete choice experiment. BMJ Open. 2020;10(11):e041592. https://doi.org/10. 1136/bmjopen-2020-041592.

12. Muqattash R, Niankara I, Traoret RI. Survey data for COVID19 vaccine preference analysis in the United Arab Emirates. Data Brief. 2020;33:106446. https://doi.org/10.1016/j.dib.2020. 106446. 
13. Liao Q, Ng TWY, Cowling BJ. What influenza vaccination programmes are preferred by healthcare personnel? A discrete choice experiment. Vaccine. 2020;38(29):4557-63. https://doi.org/10. 1016/j.vaccine.2020.05.012.

14. Genie MG, Loría-Rebolledo LE, Paranjothy S, Powell D, Ryan M, Sakowsky RA, et al. Understanding public preferences and trade-offs for government responses during a pandemic: a protocol for a discrete choice experiment in the UK. BMJ Open. 2020;10(11):e043477. https://doi.org/10.1136/bmjop en-2020-043477.

15. Provisional death counts for coronavirus disease 2019 (COVID19). National Center for Health Statistics, Centers for Disease Control and Prevention. 2020. Accessed 21 Dec 2020.

16. Pfizer and BioNTech. Pfizer and BioNTech announce vaccine candidate against COVID-19 achieved success in first interim analysis from phase 3 study. New York, USA; Mainz, Germany: Business Wire; 2020.

17. Craig BM, Rand K. Choice defines QALYs: a US valuation of the EQ-5D-5L. Med Care. 2018;56(6):529-36. https://doi.org/10. 1097/mlr.0000000000000912.

18. McFadden D. Conditional logit analysis of qualitative choice behavior. In: Zarembka P, editor. Frontiers in econometrics. Academic Press; 1974. p. 105-42.

19. de Bekker-Grob EW, Donkers B, Bliemer MCJ, Veldwijk J, Swait JD. Can healthcare choice be predicted using stated preference data? Soc Sci Med. 2020;246:112736. https://doi.org/10.1016/j. socscimed.2019.112736.

20. Poulos C, Johnson FR, Krishnarajah G, Anonychuk A, Misurski D. Pediatricians' preferences for infant meningococcal vaccination. Value Health. 2015;18(1):67-77.

21. Calman KC, Royston G. Personal paper: risk language and dialects. BMJ. 1997;315(7113):939-42.

22. Risk Science Center and Center for Bioethics and Social Sciences in Medicine. Iconarray.com. University of Michigan; 2020.

23. ChoiceMetrics N. 1.1. 1 User Manual \& Reference Guide, Australia. 2012.
24. De Moivre A. The doctrine of chances: or, A method of calculating the probabilities of events in play. Chelsea Publishing Company; 1756.

25. Schmitz C. LimeSurvey: An open source survey tool. LimeSurvey Project Hamburg, Germany URL http://www.limesurvey.org. 2012.

26. Johnson FR, Yang J-C, Reed SD. The internal validity of discrete choice experiment data: a testing tool for quantitative assessments. Value Health. 2019;22(2):157-60.

27. Jacquemet N, Luchini S, Shogren J, Watson V. Discrete choice under Oath2019 contract no.: halshs-02136103f.

28. Huff C, Tingley D. "Who are these people?" Evaluating the demographic characteristics and political preferences of MTurk survey respondents. Res Polit. 2015;2(3):2053168015604648.

29. Craig BM, Reeve BB, Brown PM, Cella D, Hays RD, Lipscomb $\mathrm{J}$, et al. US valuation of health outcomes measured using the PROMIS-29. Value Health. 2014;17(8):846-53.

30. Craig BM, Rand K, Bailey H, Stalmeier PF. Quality-adjusted life-years without constant proportionality. Value Health. 2018;21(9):1124-31.

31. Craig BM, Rand K. Choice defines QALYs: a US valuation of the EQ-5D-5L. Value Health. 2018;21:S12.

32. Stata/MP 14.2 for Windows (64-bit $\mathrm{x} 86-64)$. College Station, Texas, USA: StatCorp LLC; 2018.

33. Hollin IL, Craig BM, Coast J, Beusterien K, Vass C, DiSantostefano R, et al. Reporting formative qualitative research to support the development of quantitative preference study protocols and corresponding survey instruments: guidelines for authors and reviewers. Patient. 2020;13(1):121-36. https://doi.org/10.1007/ s40271-019-00401-x.

34. Anderson RM, Vegvari C, Truscott J, Collyer BS. Challenges in creating herd immunity to SARS-CoV-2 infection by mass vaccination. Lancet. 2020;396(10263):1614-6. https://doi.org/10.1016/ S0140-6736(20)32318-7. 